\title{
Évaporation isotherme et isobare des saumures du sud tunisien suivie par la méthode DPAO
}

\author{
H. Hammi ${ }^{1}$, J.A. Musso ${ }^{2}$, A. M'Nif ${ }^{1}$ et R. Rokbani ${ }^{1}$ \\ ${ }^{1}$ Laboratoire des Procédés Chimiques, Institut National de Recherche Scientifique et \\ Technique, BP. 95, 2050 Hammam-Lif, Tunisie \\ ${ }^{2}$ Laboratoire de Matériaux et Microélectronique de Provence, L2MP, Faculté des \\ Sciences et Techniques, Université de Toulon et du Var, BP. 132, 83957 La Garde \\ Cedex, France
}

\begin{abstract}
Evaporation is the most important process to recover salts from Tunisian natural brines. So it's essential to visualize the geometrical representations of the system involved in this case $\mathrm{Na}^{+}, \mathrm{K}^{+}$, $\mathrm{Mg}^{2+} / \mathrm{Cl}^{-}, \mathrm{SO}_{4}{ }^{2-}-\mathrm{H}_{2} \mathrm{O}$. Those representations allow us to navigate through the diagram and to draw crystallisation paths. Many techniques have been successfully tested over the last century such as the van't Hoff projection and the Janecke one, and were used to resolve the complexity of the multicomponent problem. The DPAO is a computer based visualization method proposed to enable a simulation of brine evaporation and to foresee the solid phases deposed during the process.
\end{abstract}

\section{INTRODUCTION}

Le cheminement est la suite de transformations chimiques induites par la perte ou l'ajout d'un constituant à un système d'équilibres de phases donné. Il permet de définir au cours de l'évolution du système : le nombre, la nature, la composition et la quantité relative des différentes phases condensées qui précipitent ou disparaissent. Dans notre étude, le constituant qui disparait, est l'eau, qui part sous forme de vapeur à pression et température constantes.

La représentation graphique du cheminement sur le diagramme de phases renseigne sur la suite des réactions qui se produisent lors de la variation d'une variable intensive (pression, température, composition) liée au système d'équilibres. Les bilans de matière permettent de quantifier les phases d'un équilibre.

\section{PRINCIPE DU CHEMINEMENT}

\subsection{Méthode conventionnelle}

En adoptant la représentation de Janecké [1] à partir des données bibliographiques compilées par D'Ans [2] et en appliquant les équations suivantes on aura pour le point représentatif de la saumure de Sebkha El Melah de Zarzis à $25^{\circ} \mathrm{C}$ le cheminement représenté sur les figures 1 et 2 .

$$
\begin{aligned}
& \% K_{2}=\frac{\left[K_{2}\right]}{D} \times 100 \\
& \% M g=\frac{[M g]}{D} \times 100 \\
& \% S O_{4}=\frac{\left[S O_{4}\right]}{D} \times 100 \\
& \mathrm{D}=\left[\mathrm{K}_{2}^{2+}\right]+\left[\mathrm{Mg}^{2+}\right]+\left[\mathrm{SO}_{4}^{2-}\right]
\end{aligned}
$$


Tableau 1 : Coordonnées du point $\boldsymbol{z}$ représentatif de la saumure de Sebkha El Melah de Zarzis sur le diagramme à $25^{\circ} \mathrm{C}$ :

\begin{tabular}{|c|c|c|c|}
\hline Coordonnées & $\% \mathrm{~K}_{2}$ & $\% \mathrm{Mg}$ & $\% \mathrm{SO}_{4}$ \\
\hline$z$ & 3,794 & 84,57 & 11,64 \\
\hline
\end{tabular}

En analysant ce chemin de cristallisation théorique on peut distinguer cinq séquences de cristallisation différentes par la nature des sels récupérés :

$1^{\text {ère }}$ séquence $\left(z z^{\prime}\right): \mathrm{NaCl}$ (la saumure s'évapore et dépose du $\mathrm{NaCl}$ sans changer de position ; $z$ et $z$ ' sont superposés ).

$2^{\text {ème }}$ séquence $\left(z^{\prime} x\right)$ : Halite $(\mathrm{NaCl})+$ hexahydrite $\left(\mathrm{MgSO}_{4} \cdot 6 \mathrm{H}_{2} \mathrm{O}\right)$.

$3^{\text {ème }}$ séquence (x Y) : Halite + hexahydrite + Kaïnite $\left(\mathrm{MgSO}_{4} \cdot \mathrm{KCl}_{3} 3 \mathrm{H}_{2} \mathrm{O}\right)$.

$4^{\text {ème }}$ séquence $(\mathrm{Y} \mathrm{R})$ : Halite + Kiesérite $\left(\mathrm{MgSO}_{4} \cdot \mathrm{H}_{2} \mathrm{O}\right)+$ Kaïnite.

$5^{\text {ème }}$ séquence $(\mathrm{R} \mathrm{Z})$ : Halite + Kiesérite + Carnallite $\left(\mathrm{MgCl}_{2} \cdot \mathrm{KCl} \cdot 6 \mathrm{H}_{2} \mathrm{O}\right)$.

Le chemin obtenu est conforme aux règles de Berthon [3], en effet le point figuratif de la solution étudiée évolue du point de départ $z$ jusqu'à ce qu'il arrive au point $Z$, point final de cristallisation en parcourant les domaines de l'hexahydrite $\left(\mathrm{MgSO}_{4} \cdot 6 \mathrm{H}_{2} \mathrm{O}\right)$, de la Kaïnite $\left(\mathrm{MgSO}_{4} \cdot \mathrm{KCl} .3 \mathrm{H}_{2} \mathrm{O}\right)$ et de la carnallite $\left(\mathrm{MgCl}_{2} \cdot \mathrm{KCl} 6 \mathrm{H}_{2} \mathrm{O}\right)$ tout en restant dans le domaine saturé en $\mathrm{NaCl}$ qui se dépose avec toutes les séquences de cristallisation.

Le chemin réel obtenu suite à l'évaporation isotherme est différent du chemin théorique. il est possible de dégager 3 types de sels répertoriés dans le tableau I.

Tableau 2 : Répartition des sels récupérés expérimentalement par évaporation isotherme à $25^{\circ} \mathrm{C}$ de la saumure naturelle de Sebkha El Melah de Zarzis.

\begin{tabular}{|c|c|}
\hline Désignation & $\mathbf{2 5}^{\circ} \mathbf{C}$ \\
\hline Sel 1 & $\begin{array}{c}1,241<\text { densité }<1,292 \\
\text { NaCl }\end{array}$ \\
\hline Sel 2 & $\begin{array}{c}1,292<\text { densité }<1,310 \\
\text { NaCl }+ \text { sulfates simples ou doubles. }\end{array}$ \\
\hline Sel 3 & $\begin{array}{c}1,310<\text { densité }<1,340 \\
\mathrm{NaCl}+\text { Carnallite }+ \text { sels magnésiens sulfatés. }\end{array}$ \\
\hline
\end{tabular}

Malgré la différence constatée entre les chemins théorique et expérimental, on a dégagé une similitude entre les sels récupérés et prévisibles.

En effet le sel $\mathrm{N}^{\circ} 1$ et la $1^{\text {ere }}$ séquence ainsi que le sel $\mathrm{N}^{\circ} 3$ et la $5^{\text {ème }}$ séquence sont parfaitement comparables. Il s'agit de $\mathrm{NaCl}$ dans le $1^{\text {er }}$ cas et de Carnallite dans le second. Les séquences 2,3 et 4 correspondent au sel $\mathrm{N}^{\circ} 2$. 


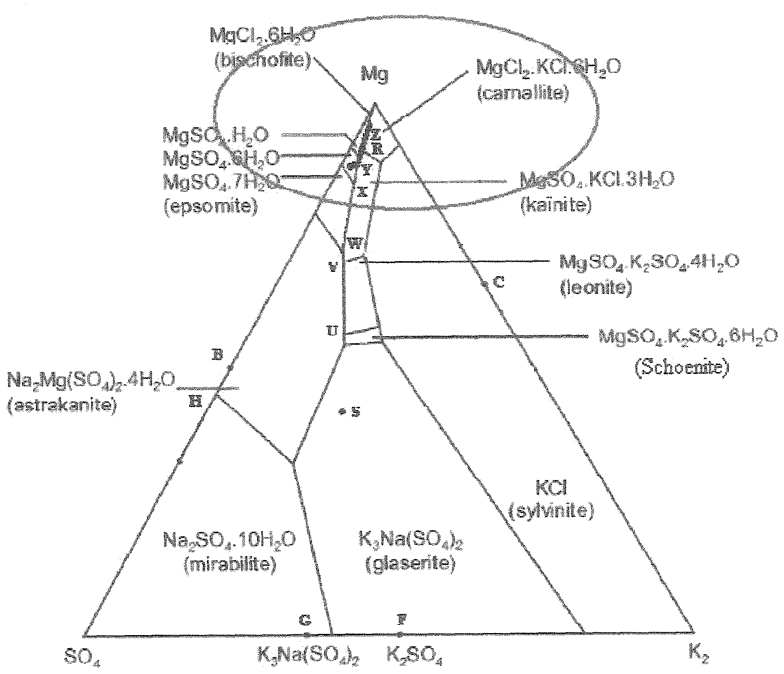

Figure 1 : Cheminement théorique du point représentatif de la saumure de Sebkha El Melah de Zarzis

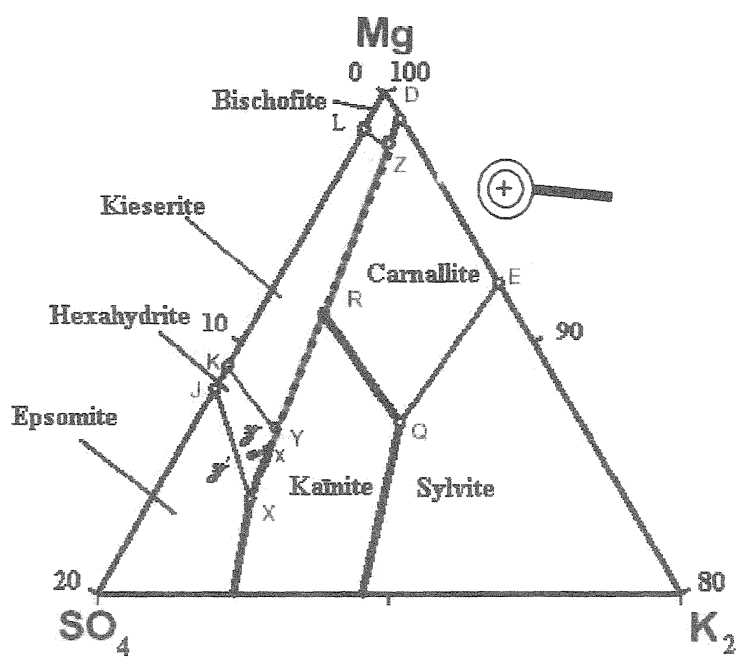

Figure 2 : Agrandissement de la partie où évolue le point représentatif de la saumure de Sebkha El Melah de Zarzis 


\subsection{Méthode DPAO (Diagramme de Phases Assisté par Ordinateur)}

Les données nécessaires à l'établissement d'un cheminement sont, pour chaque point, leurs coordonnées et la nature des phases en équilibre, et, pour tous les domaines de variance, leurs limites déterminées par itération à partir des limites des domaines de variances inférieures. Ces données ont été établies automatiquement par le premier logiciel de la méthode DPAO "Diagramme de Phases Assisté par Ordinateur» [4] dans l'hyper-espace de tous les constituants.

A chaque étape du cheminement, il est nécessaire de connaître la composition du liquide en équilibre avec les phases solides. Ces résultats sont obtenus à partir de procédures élémentaires du second logiciel de DPAO[5,6]. Divers types de procédures sont utilisés : bilan de matière, cheminements dans des domaines de variance différents, décision aux points invariants, tests d'arrêt du cheminement.

\subsubsection{Le bilan de matière}

L'évaporation isotherme et isobare est contrôlée à chaque étape par la nature de la réaction chimique qui peut avoir lieu dans chaque domaine de variance. Nous schématiserons cette réaction par le bilan de matière suivant :

$$
\left.\mathbf{q}_{\mathbf{0}}\left|\mathbf{L}_{\mathbf{0}}\right| \rightarrow \mathbf{q}|\mathbf{L}|+\Sigma \mathbf{q}_{\mathrm{i}} \mid \text { solide(s) }\right)_{\mathrm{i}}\left|+\mathbf{q}_{\mathrm{v}}\right| \mathbf{H}_{\mathbf{2}} \mathbf{O}_{\mathbf{v}} \mid
$$

Les vecteurs $\left|\mathrm{L}_{0}\right|,|\mathrm{L}|, \mid$ solide $(\mathrm{s})_{\mathrm{i}} \mid$ et $\left|\mathrm{H}_{2} \mathrm{O}_{\mathrm{v}}\right|$ représentent respectivement les compositions (variables intensives) du liquide initial, du liquide à l'étape analysée, des solides en équilibre et de l'eau sous forme de vapeur; les variables $\mathrm{q}_{0}, \mathrm{q}, \mathrm{q}_{\mathrm{i}}$ et $\mathrm{q}_{\mathrm{v}}$ représentent respectivement les quantités (variables extensives) de ces mêmes espèces.

Le nombre de solides en équilibre dépend, évidemment, de la variance du domaine dans lequel a lieu la réaction.

\subsubsection{Cheminement dans des domaines}

Plusieurs types de cheminement sont traités :

- Cheminement dans le domaine des liquides non saturés ;

- Cheminement dans le domaine de saturation en un ou plusieurs sels ;

- Cheminement sur une nappe de cristallisation d'un ou plusieurs sels ;

- Cheminement sur une ligne mono-variante de précipitation d'un ou plusieurs sels.

Dans chacun de ces types de cheminement la cote en eau est diminuée d'une infime quantité, le bilan matière est établi et l'analyse d'atteinte d'une frontière du domaine est effectuée.

\subsubsection{Actions aux frontières}

Plusieurs types de décision sont analysés. Ce sont la composition des phases en présence et la géométrie du diagramme qui vont orienter le cheminement :

- Deux sels sont les hydrates différents d'un même sel : la déshydratation du sel le plus hydraté a lieu, sans parcours d'un domaine.

- $\quad$ Aucun sel n'est l'hydrate d'un autre sel : c'est la position relative des trois lignes qui concourent au point invariant qui détermine la suite du cheminement. 
- Les points limites des trois lignes ont une cote en eau supérieure à celle du point invariant : le point final de cristallisation par évaporation est atteint.

- Les points limites de deux lignes ont une cote en eau supérieure à celle du point invariant : la troisième ligne est parcourue.

- Le point limite d'une seule ligne a une cote en eau supérieure à celle du point invariant : la nappe limitée par les deux autres lignes est parcourue.

\subsubsection{Arrêt du cheminement :}

Deux types d'arrêt du cheminement sont prévus :

- Arrêt par épuisement du liquide ;

- Arrêt lorsque le point de plus basse cote en eau dans le diagramme est atteint.

Ces deux cas correspondent a l'impossibilité de poursuivre l'évaporation.

\section{CONCLUSION}

A partir des résultats précédents et ceux du cheminement d'un point mélange représentant la saumure naturelle de Sebkha El Melah de Zarzis, il est possible d'établir une comparaison entre le cheminement prévu par ordinateur et celui conduit expérimentalement dans les conditions proches de celle du site et de dégager les similitudes entre les deux approches, le but étant la vérification de l'utilisation du diagramme de phase assisté par ordinateur pour prévoir quantitativement les phases solides récupérables. En cas de résultat concluant il serait possible d'appliquer cette méthode à l'étude de l'évaporation isotherme de toutes les saumures contenues dans les Sebkhas et Chotts du Sud Tunisien qui sont assimilées au même système quinaire océanique $\mathrm{Na}^{+}, \mathrm{K}^{+}, \mathrm{Mg}^{2+}, \mathrm{Cl}^{-}, \mathrm{SO}_{4}{ }^{2-} / / \mathrm{H}_{2} \mathrm{O}$ et ne diffèrent que par leur composition initiale.

\section{Références}

1. E.Janecke, Z.anorg.allgem.Chem.,53, 319 (1907)

2. J.D'ans in Die Losungsgleichgewichte der Systeme der Salze Ozeanischer Salzablagerungen 2545 Kali-Forschung sanstalt.Verl. ges. F. Ackerbau 1933.

3. R. Berthon, in Représentation des équilibres de solubilité et utilisation des diagrammes, Paris. 1962, Gauthier-Villars éditeur. p 237-241.

4. J.A. Musso in Diagramme de phases assisté par ordinateur : représentation et cheminement isothermes et isobares. Thèse d'état Université de Toulon et du Var 1989.

5. J.A. Musso : Solubility Phase Diagrams Coupled to Computer Science (DPAO)

Part I : Theory of the Sequential Representation; CALPHAD, 27( 1), 65-69 (2003).

6. H.Hammi; J.A. Musso; A.M'Nif; R.Rokbani: Solubility Phase Diagrams Coupled to Computer Science (DPAO) Part II : Applied to Isothermal Evaporation of Tunisian Natural Brines; CALPHAD, 27( 1), 70-77 (2003). 
